
\title{
ZS Research Square \\ Virtual Cleaning of Works of Art Using Deep Convolutional Neural Networks
}

\author{
Morteza Maali Amiri ( $\nabla$ mm2391@rit.edu ) \\ Rochester Institute of Technology https://orcid.org/0000-0002-0391-3310 \\ David W Messinger \\ Rochester Institute of Technology
}

\section{Research Article}

Keywords: Virtual cleaning, Convolutional Neural Network, Varnished and unvarnished artworks

Posted Date: June 4th, 2021

DOl: https://doi.org/10.21203/rs.3.rs-232574/v1

License: (9) This work is licensed under a Creative Commons Attribution 4.0 International License. Read Full License

Version of Record: A version of this preprint was published at Heritage Science on August 3rd, 2021. See the published version at https://doi.org/10.1186/s40494-021-00567-4. 


\title{
Virtual Cleaning of Works of Art Using Deep Convolutional Neural Networks
}

\author{
Morteza Maali Amiri and David W Messinger \\ Rochester Institute of Technology \\ Chester F Carlson Center for Imaging Science, \\ 54 Lomb Memorial Drive \\ Rochester, NY 14623 \\ mm2391erit.edu
}

\begin{abstract}
Virtual cleaning of art is a key process that conservators apply to see the likely appearance of the work of art they have aimed to clean, before the process of cleaning. It is also of public interest, allowing people to see their favorite work of art without the yellow tint of the varnish which has covered the work. There have been many different approaches to virtually clean artwork, all of which need to physically clean the work in at least a few spots, an impediment in some cases. Another issue regarding the methods devised until now is that some of them need samples of pure white or black paint on the painting which might be, again, an obstacle as there are certainly works without any pure black and white paint. To overcome these shortcomings, a Convolutional Neural Network is trained on representative RGB images; the images are first artificially yellowed using a physics based model and the network is trained to go from the yellowed images to the original colored images. The results show improvement over a proposed method using a physical model to remove the impacts of age. The CNN is then applied to images of the Mona Lisa and The Virgin and Child with Saint Anne, both painted by Leonardo da Vinci, and works for which we have images from both before and after physical cleaning. Results show both a qualitative and quantitative improvement in the color quality of the resulting image. The novelty of the work proposed herein lies in two premises. First is the accuracy of the method, which is demonstrated through comparing the method with the only physical approach derived until now. Second is the generalizability of the method which is shown through blindly applying the method to two famous works of art for which no information but an RGB image of the uncleaned artwork is known.
\end{abstract}

Keywords: Virtual cleaning, Convolutional Neural Network, Varnished and unvarnished artworks

\section{Introduction}

It is a well-known physical phenomenon that the appearance of varnish on the surface of a painted work of art will change over time, altering the visual qualities of the work. There are artists who did not intend for their paintings to be varnished but once the paintings were out of their hands, the artworks were varnished mostly for protection purposes [1-3]. Although effective in protecting the artwork from dirt and pollutant, varnish application can substantially change the appearance of paintings [4], particularly after the passage of a significant amount of time. The change of the appearance of the painting due to varnishing depends on many factors, the most important of which are the type of varnish (i.e., its molecular weight) and the age of the varnish $[4,5]$. Artwork cleaning is considered one of the most significant duties undertaken by conservators due to the irreversibility of the action. Cleaning is comprised of removal of undesired deposits as well as the aged varnish from the surface, which helps reestablish the original appearance of the painted surface [6-8]. There are two main approaches that have been taken to "clean" works of art, physical and virtual. The physical approach, in which mild solvents and gel systems are usually used, apart from being time consuming, can also damage the work [9-11]. The simulation of the outcome of the aged varnish removal from a painting is referred to as virtual cleaning. Virtual cleaning supplies the conservators with a representation of the appearance change that would likely be achieved should the cleaning process be undertaken. Additionally, in some cases where the painting is not likely to go through removal of varnish sometime soon, the simulation becomes even more important [12]. 

mentioned here. Barni et al. (2000) [13] developed an image processing technique to virtually clean artwork. In their work, first they physically cleaned a small part of the painting; subsequently, they found the matrix transform in the RGB domain between the cleaned part and the corresponding uncleaned part from the artwork. They then applied the same matrix transformation to the uncleaned parts of artwork and were able to virtually clean the entire piece. Papas and Pitas (2000) developed a function to recover the cleaned version of a work of art from the uncleaned one using a few different approaches [14]. They noted that using the RGB color space of the camera is not suitable as it does not closely correlate with human perception of color. Therefore, they used the CIELAB color space in their work claiming it performs better due to its higher visual uniformity [14]. They also had to first physically clean the artwork in a few regions; they subsequently used the mean values of those regions in both the cleaned and the corresponding uncleaned image. They developed several different transfer functions, of which they showed that their linear approximation (which is based on a linear transformation from a varnished to a cleaned image) and white point (which is based on the chromatic adaptation taken from color science) have outperformed others [14]. Elias and Cotte (2008) were able to virtually clean the famous Mona Lisa through having access to the paints used by Leonardo da Vinci at the time of painting the work of art [15]. To do this, a color chart made out of classical paints utilized in 16th century in Italy were made in varnished and unvarnished forms. Using these charts, they were able to deduce a mean multiplicative factor for each wavelength. The factor was then applied to the Mona Lisa's spectra leading to the virtual removal of the varnish [15]. Palomero and Soriano (2011) came up with a neural network to approach the issue of virtual cleaning for the first time [16]. They trained a shallow neural network with 2 hidden layers and 30 neurons to go from varnished painting to the unvarnished one. They again had to physically clean a part of the painting. RGB data of the cleaned and the corresponding uncleaned region of the painting were used to train the network. Using estimation methods they were able to also estimate the spectral reflectance of the varnish layer, supposing that the varnish acts as filter over painting.

Trumpy, et al. (2015) were the first to attempt to approach the virtual cleaning of artwork using a completely physical model for the varnish/painting system [12]. They developed the first physical approach attempting to model the effect of varnish and obtain the spectra of the cleaned artworks. They started with the Kubelka-Munk theory [17] and developed a formula that predicts the spectra of the cleaned painting. To obtain the cleaned spectra they needed to first estimate the varnish transmittance and for that, they made a few simplifying assumptions such as that a dark site of the painting consists of a perfect black and absorbs all the incident radiation. They also assumed that the varnish spectral reflectance is not wavelength dependent. Through physically cleaning the black and white parts (assuming the painting has pure black and white paints) of the painting they were able to estimate the spectral transmittance of the varnish [12]. This model is of great importance to us, as it is the first work trying to lay out a basis model for the varnish/painting system and it is used as a reference to compare our results with the model derived by the authors in [12].

Later on, Kirchner, et al. (2018) developed a method based on the Keubelka-Munk theory attempting to virtually clean artwork [18]. In order to do that, they had to physically remove the varnish from parts of the painting and measure the spectral reflectance before and after varnish removal. One of the key measurements was to measure the pure white on the painting through which they were able to compute the transmittance and reflectance of the varnish layer using two constant KubelkaMunk theory. After characterization of the varnish layer, they were able to digitally clean the full painting [18]. Linhares, et al. (2020) used hyperspectral imaging to first measure the spectra of two paintings before and after varnish removal. Using this information they were able to characterize the varnish layer which subsequently allowed for virtual removal of the varnish layer [19]. The need to specify the pure black and white, physical removal of the varnish from the painting, use of spectral reflectance and the inability to generalize the method and results to other works are only a few shortcomings of the works reported here.

The purpose of this work is to address the shortcomings of the prior methods and find a better approach to virtually clean the artwork fulfilling both public and conservator's interests in easily seeing how an old, varnished painting would visually appear without the varnish layer. In order to do that, the virtual cleaning was approached using convolutional neural networks (CNNs), a technique termed also deep learning. CNNs have been applied in different areas, particularly in machine learning to solve different problems in the image processing [20-23]. Of special interest to us is image colorization using deep learning [24-27]. Image colorization refers to the process that changes black and white image into a colored image trying to recover the original colored scene, especially from old black and white pictures. This process inspired us to use CNNs to go from varnished work of art (a yellowed image) to an unvarnished one (a colored image). To do that, images of rural and urban areas, people, and color charts, were artificially yellowed either in the spectral domain which is done through the light interaction with varnish/paint system, or in RGB color domain, which is done through multiplying a yellow filter by the images point by point. The CNN is trained to go from the yellowed images to the original colored images. The paper reported by [12] is used as a reference and our results are compared to theirs. Comparison of our results to the paper proposed 
by [12] shows that the method proposed herein has outperformed the physical method. Two famous artworks, namely the Mona Lisa and The Virgin and Child with Saint Anne, both painted by Leonardo da Vinci, were also fed into the trained network, resulting in cleaned versions of both. Comparison between our results and the cleaned versions of the artworks shows that our trained network has an ability to predict the cleaned version of works of art even without being trained on any similar paintings. Using the method proposed here, there is no need to physically remove the varnish and measure the spectral information of the painting; the need to know where the pure black and white paints are located is also erased. This approach also has the potential to be generalized to many types of artwork, given an appropriate training data set.

This paper is laid out in the following manner. Section 2 describes the data sets used in the work, the convolutional neural network proposed, and the experiments performed. Section 3 presents the results and discussions in which the results of our method are going to be compared with the physical approach proposed by [12] along with the results obtained from applying the method to the Mona Lisa and The Virgin and Child with Saint Anne. We end our paper with conclusions summarizing the paper's contributions and the outcomes.

\section{Methodology}

This section first describes the data used to train and test the algorithm. The two major parts, namely, spectral and color simulations are expanded upon and described. The criteria used to evaluate the success of the method proposed here are also presented and explained.

\subsection{Data}

In this work, the problem of virtual cleaning of works of art is looked upon as a machine learning problem, in which a system (a CNN herein) learns to go from an uncleaned artwork to a virtually cleaned one. The use of a CNN approach for this problem requires a set of both training and testing data for the learning process. Here, the hypothesis is that the training data does not need to be a combination of cleaned and uncleaned works of art, but instead can be images of similar content (i.e., people, landscapes, buildings, etc.) in both "cleaned" and "yellowed" states. As described below, the "yellowed" data is simulated based on a physical model of the spectral impacts of an aged varnish. To train the networks, urban and rural images taken from the Kaggle website [28], along with images of people and color charts (described below) were used to train the CNN. Some of the images are shown in Fig. 1. We add the color charts as they represent a large range of colors which might not have been represented in the other images. To ensure our training data covers a wide range of colors, the color charts are simulated and added to the dataset as well.

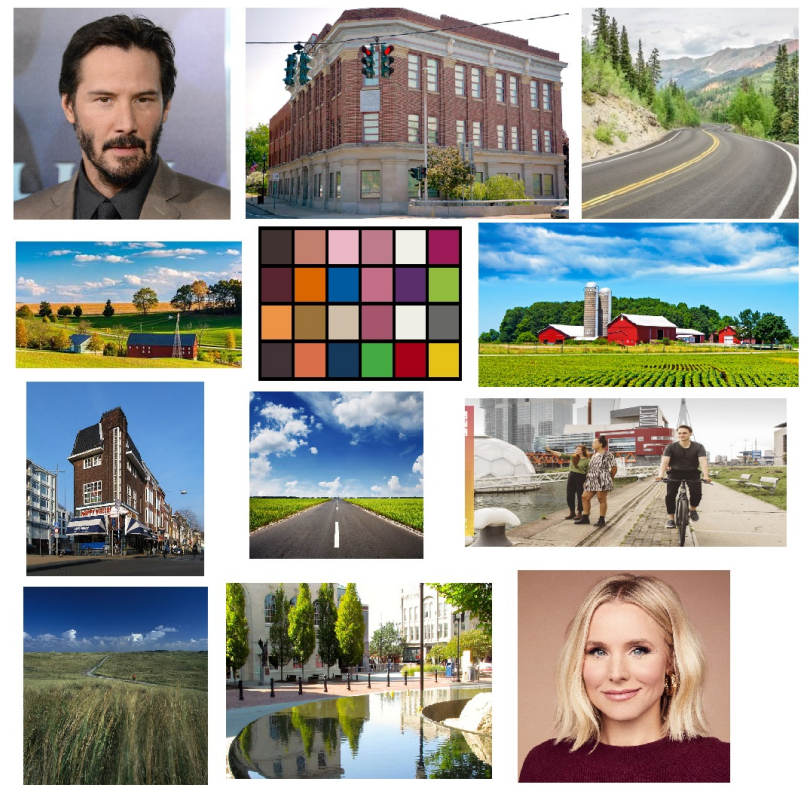

Figure 1. Some examples of the images used for training in this work.

It should be noted that the color charts are simulated using 1269 spectral reflectances of Munsell chips from the Munsell Book of Color Matt Finish Collection [29], 264 spectral reflectances of ANSI IT8.7/2 Standard Chart from Kodak, 1950 

Macbeth ColorChecker. All of the samples were simulated into a 4 by 6 color chart the same as the Macbeth ColorChecker with 24 chips. To make the simulated color charts, the spectral reflectances were first transformed to CIEXYZ using standard formulae and then the CIEXYZ was transformed to sRGB. The final images were saved in jpg format [30]. There are 154 simulated color charts overall and 488 images of people, urban, and rural areas.

As it is described later in section 2.2, there are two major parts to this work. The first is called spectral simulation, in which only the color charts are used. The second part is called color simulation in which the 488 images of urban and rural areas are combined with 22 images of the color charts. The color charts are chosen based on the different number of colors they contain. The more different colors they have, the higher their likelihood to be picked out to be one of the 22 images. As mentioned, the reason to use the color charts is they represent different colors that might not be found in the other images in our dataset, therefore, we did our best to pick the color charts that represent colors that are widely diverse.

\subsection{Procedure}

This paper aims at finding a better way to virtually clean artwork which allows everyone, including both people that are interested in arts and conservators, to readily see how an old, varnished painting will appear without varnish, referred to as virtual cleaning of works of art. To do this, a convolutional neural network is proposed. The proposed method is trained to go from yellowed "varnished" images to color "unvarnished" images of artworks. The varnished and unvarnished artworks are simulated either in the spectral domain, under the section called spectral simulation, or in the RGB color domain, under the color simulation, both explained below. The reason for using these titles is to first be able to compare our results to the physical model, in which the works of art are virtually cleaned in the spectral domain. Thus, we also simulate our artworks in the spectral domain. After spectral simulation, to generalize the method to more works of art, RGB color simulation of works of art is used, in which there is no need to know any information and accessing RGB data of the uncleaned work of art suffices. In other words, the color simulation section shows the applicability of the method proposed herein to famous artworks (i.e., any artworks), for which cleaning is simply not possible either because of the expense of the artwork or not having access to any cleaned parts of the work, and having access to one RGB image of the uncleaned work is the only data available. Comparison with the physical method for the color simulation section is simply not possible as the physical model needs at least the paintings to be cleaned at the brightest and darkest parts.

\subsubsection{Spectral Simulation}

As mentioned above, spectral simulation here refers to simulating cleaned and uncleaned works of art in the spectral domain, aligning with the physical model proposed by [12]. They were the first to attempt to physically model the relationship between the painting and the varnish. We refer to their model as the physical model from now on. The physical model they presented is in the spectral domain that was obtained using a meticulous step by step analysis of the interaction between light and the varnish/painting system. They based their main equation on Kubelka, and were able to derive a model that enabled them to obtain the reflectance spectra of the cleaned work of art from an uncleaned one. One of the unknowns in this model is the transmittance of the varnish. Assuming that there are perfect black and white spots on the artwork, they estimated the transmittance using the cleaned and uncleaned black and white reflectance spectra. Therefore, they were able to use the model to predict the uncleaned spectra of the work of art. For us to be able to draw a fair comparison between our model and theirs, we should also do the simulation in the spectral domain with the light matter interaction model in mind. To do that, we consider the Macbeth ColorChecker as a simulated "work of art". The reason for choosing Macbeth is it has pure black and white spectra along with other colors. The physical model relies on first finding the pure black and white parts of the painting, therefore, using a Macbeth ColorChecker is justified.

To simulate the interaction of light with varnish/painting system, Fig. 2 is used as a guide. Here, $R_{t}, R_{V}$, and $R_{P}$ denote the spectral reflectances of the uncleaned artwork (or the total effective reflectance of the varnished work of art), varnish and the paint, respectively. $T$ also denotes the transmittance spectra of the varnish. It should be noted that $R_{P}$ here is the Macbeth ColorChecker. It goes without saying that the cleaned spectra is $R_{P}$. The measurement geometry is $45 / 0$ as it was the case in [12] as well. It should be noted that 45/0 refers to the method of measurement (illumination/viewing) in which the illumination is at 45 degrees off axis and the observer is at 0 degrees. Using the same approach as [12], the transmittance and the reflectance spectra of the varnish are presumed to be as shown in Fig. 3.

In general, the varnish is yellow and the transmittance and reflectance spectra of the varnish should represent that [12,18]. The reflectance of the varnish is very low as [12] assumed that the body reflection of the varnish is equal to the black reflectance spectra covered with varnish (or $R_{t}$ measured over a dark area). Here, we use the Macbeth ColorChecker as a 


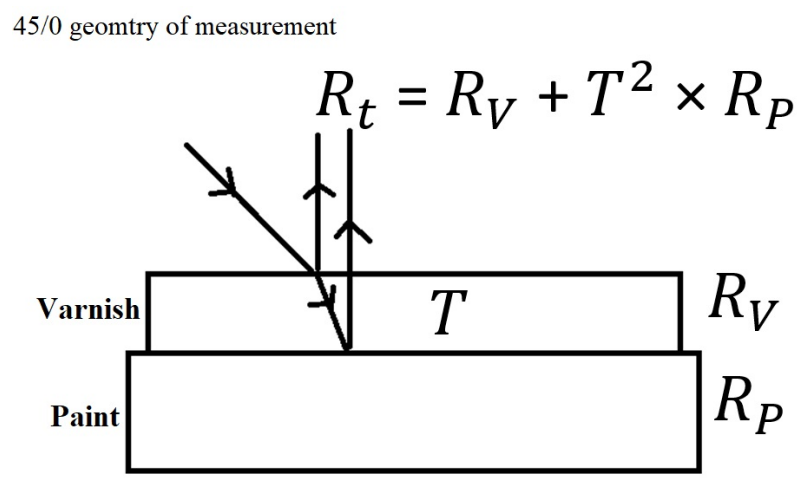

Figure 2. Interaction of light with varnish/painting system.

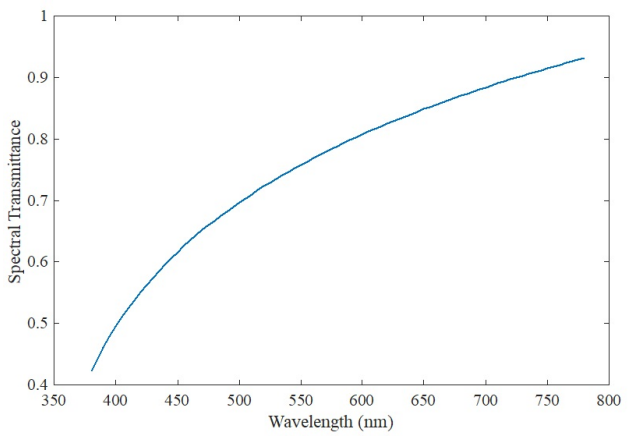

(a)

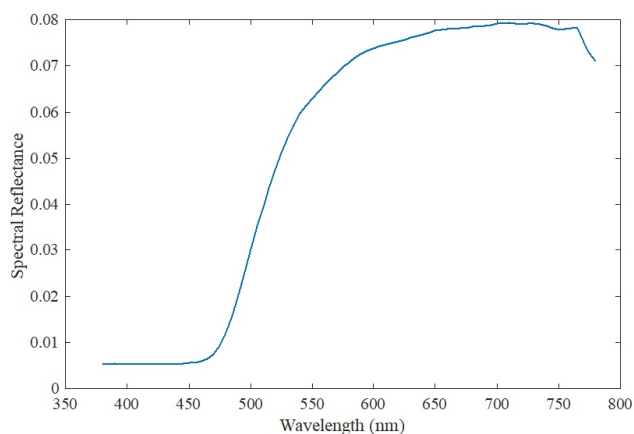

(b)

Figure 3. (a) Transmittance and (b) reflectance spectra of the varnish.

"simulated work of art" and the physical model is used to see how well it predicts the clean spectra of the Macbeth color chart.

After applying the physical model, it is time to apply the CNN model that has been devised in this paper (described below). To train the CNNs we need two sets of images, one "yellowed" set and the other set in the original color. Using 1,269 spectral reflectances of Munsell chips, 264 spectral reflectances of ANSI IT8.7/2 Standard Chart from Kodak, 1,950 samples of the Natural Color System, and 130 spectral reflectances of Artist's paint [31], 154 color charts are simulated in a way that each color chart has 24 chips in the same arrangement as in the Macbeth ColorChecker. Because we have access to the spectra of all these samples, we use the approach presented in Fig. 2 to simulate yellowed color charts as a way to simulate "varnished works of art". Therefore, using all these samples we are able to make a fairly large set of training data, using which the CNN is trained to go from yellowed images to images in their original color. After training the CNN, the Macbeth color chart is used to test the CNN and see how well the network can recover the original color chart from the yellowed one. At this point, we also compare the results to the results obtained from the physical model [12]. To make the comparison possible between the work reported herein and the physical model reported in [12], the simulation of the cleaned and uncleaned works of art should also be done in the spectral domain hence the use of spectral reflectances of Macbeth ColorChecker. The results using both the physical model and the $\mathrm{CNN}$ are visualized in the results and discussions section, along with quantitative analysis and comparison.

\subsubsection{Color Simulation}

The second part of the paper focuses on simplifying the work, so that it could be applied to any RGB image of a piece of art with the goal of virtually "cleaning" it. In other words, the simulation of the cleaned and uncleaned artworks is done in the RGB color domain hence the use of color simulation for this section. In order to do that, all the images of urban and rural areas, people, and color charts are combined to form a dataset of 500 images which are used as training data. Now because we do not have access to the spectral information of all of these images, we cannot yellow them the same way as in Section 2.2.1. Therefore, we developed yellow filters, as shown in Fig. 4, which are multiplied point by point with all the 500 images, 
resulting in the yellowed (or "varnished") images.

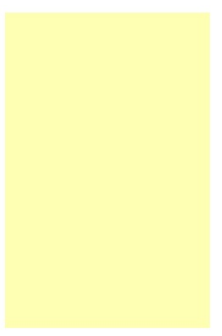

(a)

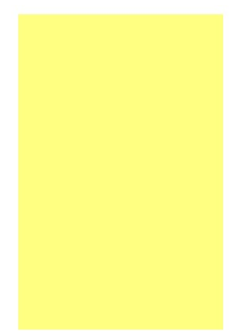

(b)

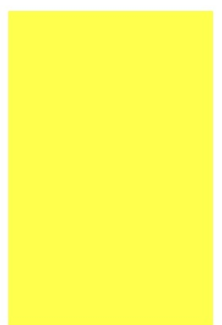

(c)

Figure 4. Yellow filters at three levels used to artificially yellow the images.

As shown in Fig. 4, three levels of this filter are used, slightly, moderately, and highly yellow, notionally representing various degrees of aging. Our aim here is to train a CNN so that it can estimate the original colored images from the yellowed samples. After training we want to apply this network to two famous works of art with images available from the internet, namely Mona Lisa and The Virgin and Child with Saint Anne, both painted by Leonardo da Vinci. An interesting point about these paintings is that their cleaned version is also available, so we can feed the varnished (uncleaned) versions through the network and compare the results to the cleaned versions as "ground truth" for evaluation. To do the training, first a judgment should be made about how yellow the filter should be; we chose the moderately and highly yellowed filter for The Virgin and Child with Saint Anne and Mona Lisa, respectively, after visually judging the cleaned and uncleaned artworks. The CNN is then trained on the yellowed and cleaned images in the training set, and after that, the artworks are fed into the network as a test to see how well the approach works. At the end, the results are compared to the physically cleaned versions of the artworks. It should be noted that the artworks are not used to train the network and they are only used to test the performance of the trained network.

\subsection{Convolutional Neural Network}

\subsubsection{Architecture}

Keras, a library written in python for the purpose of deep learning, was used in this work [32]. A deep convolutional neural network $(\mathrm{CNN})$ was built here with 11 layers. The architecture of the network is shown in Fig. 5 . As it is observed from the figure, there are 11 layers to the network used in this work. The stride 2 has also been used here leading to the down-sampling of the image, which is compensated for by using up-sampling later on in the network. In other words, the input and output have the same size, and the size of the input remains intact in output. It should be noted that the size of the image input to the network should be $400 \times 400.500$ training images of urban and rural areas along with people and color charts are used to train this network to learn how to transform a yellowed (varnished) image to the original color (unvarnished) image.

\subsubsection{Application of the CNNs}

The $\mathrm{CNN}$ used here is restricted to an input image of size $400 \times 400$ pixels. Consequently, we need to adjust the input image to fit this $\mathrm{CNN}$ architecture, while maintaining the ability to transform larger (or smaller) images. In so doing, some images become blurry when going through the CNN, especially if the size of the image is much bigger than the CNN input size. Also, the feature extraction would blur the output image from the CNN. Therefore, these two causes work together to make the output image blurrier than the input. Fortunately, we can leverage existing work from JPEG compression. In order to do that, before inputting the RGB image into the network, it is first changed into a CIELAB representation, assuming that the RGB image follows the sRGB formula, and its $\mathrm{L}^{*}$ channel is preserved and set aside. After that, the original RGB image is fed into the CNN. The output of the CNN, which is also an RGB image, is then changed to CIELAB and its a* $b^{*}$ channels are extracted. These $a^{*} b^{*}$ channels are then concatenated with the $L^{*}$ of the input image that was set aside. This new CIELAB image, which has been partially transformed by the CNN, is then changed back to RGB. Using this simple method, the sharpness of the image is maintained and the approach can be applied to images of a varying size. Fig. 6 shows this process schematically. This process is adapted from JPEG image compression which is referred to as "lossless compression", meaning one could compress the color channels significantly without noticeable changes as long as the luminance channel is left untouched [33]. 
It is important to know that this process is only applied to the test images that are the focus of the virtual cleaning. There is no need to do this for the training images. Therefore, after training the network, when the testing images are fed into the network, they go through the process shown in Fig. 6.

\subsection{Evaluation Metrics}

Visualization of the results is the first method to evaluate the success of the approaches used to tackle the problem of virtual cleaning of works of art. The spectral Euclidean distance and spectral angle (SA) were also calculated between the original (cleaned) image and the "virtually cleaned" image [34]. The spectral angle between two pixels is simply the angle between them in the color space, given by

$$
S A_{k}=\cos ^{-1}\left(\frac{\mathbf{t}_{k} \cdot \mathbf{r}_{k}}{\left|\mathbf{t}_{k}\right|\left|\mathbf{r}_{\mathbf{k}}\right|}\right)
$$

where $k$ represents the $k^{t h}$ pixel, $\mathbf{t}_{k}$ and $\mathbf{r}_{k}$ represent the two pixels belonging to the test and reference images, and $S A_{k}$ represents the spectral angle between the truth and transformed images for the $k^{t h}$ pixel. To numerically report the results over the whole image with only one numerical value, the average of $S A_{k}$ over the whole image is also reported.

\section{Results and Discussions}

This section is divided into two subsections, namely, spectral simulation and color simulation. The first subsection is devoted to comparison of our results to the physical model proposed by [12] and only the simulated color charts are used for that matter. The reason for doing so is that the physical model has been derived in the spectral domain and to simulate varnished samples of the same level so that they could be used in both physical model and the CNN, the samples are simulated in the spectral domain. The second subsection talks about the application of the method suggested in this work to two real works of art, namely, Mona Lisa and The Virgin and Child with Saint Anne. The second subsection is related to applying the method to the real artworks and trying to generalize it to a wider artworks without need to any information known about the artwork.

\subsection{Spectral Simulation}

Fig. 7 shows a few of the color charts simulated in this work along with their yellowed versions. The Macbeth ColorChecker is the one on the far right, which is used as a testing sample to the CNN model built in this work. The top row 
shows the colored ("unvarnished") charts and the bottom row shows the yellowed ("varnished") charts. The Macbeth ColorChecker is also used to test the physical model. Therefore, the same color chart with the same level of yellowness is used for both methods, making the comparison between different approaches possible.

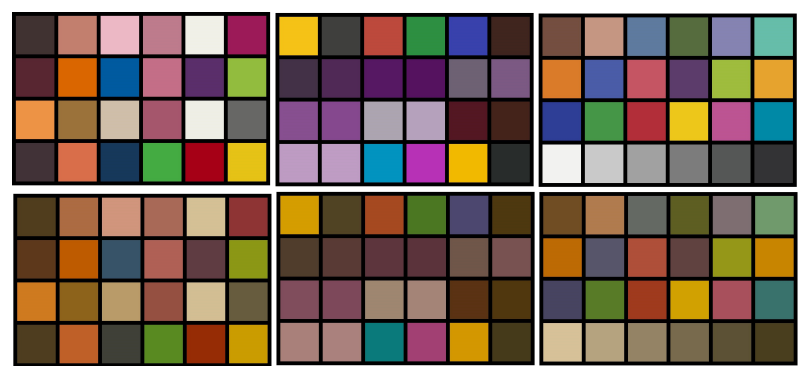

Figure 7. Simulated color charts (top row) and their yellowed versions (bottom row). 
As mentioned before, the Macbeth color chart is used as a "simulated work of art" and the results of the CNN are compared to the results obtained by the physical model proposed by [12]. In order for us to be able to compare our work with the physical model, we had no other option but to simulate the Macbeth ColorChecker and use it as an example "work of art". Fig. 8 shows the results of the method proposed here using the CNN along with the result from [12], referred to as the physical model. It should be noted that the output of the physical model is spectral reflectance; so, the reflectance was changed to CIEXYZ and then CIEXYZ was changed to sRGB. Looking at Fig. 8, it is clear that the CNN has outperformed the physical model in a
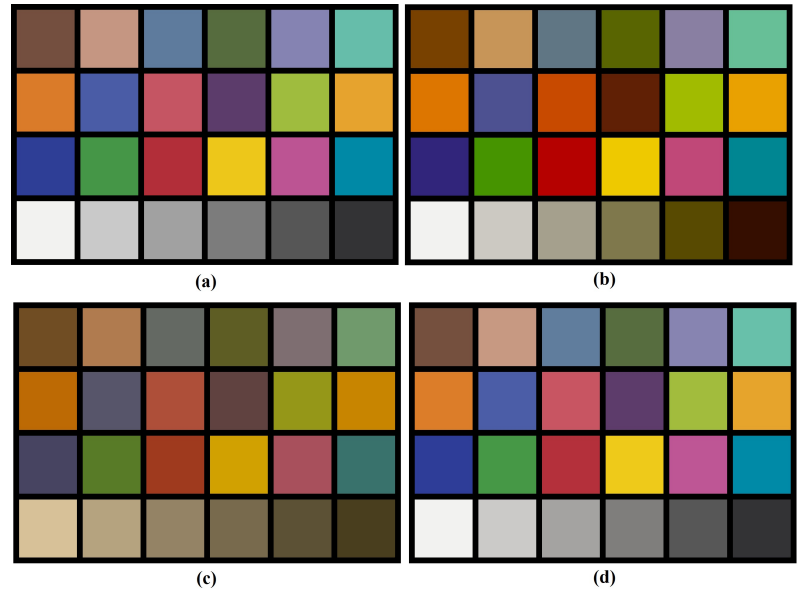

Figure 8. a: original Macbeth, b: physical model output, c: yellowed Macbeth, d: CNN output.

significant manner. To have a better understanding of the results and how they are compared in a quantifiable manner, the Euclidean distance and SA between the original Macbeth color chart (the simulated cleaned "work of art") and the output of the CNN and physical model are also calculated and shown in Table 1. It should be noted that the values reported are the averaged values across the whole image; in other words, the Euclidean distance and the SA are calculated per-pixel for the color chart and the output of CNN and the physical method and then the mean value is calculated and reported. Table 1

Table 1. Euclidean distance and SA mean values between the original and "cleaned" Macbeth color chart..

\begin{tabular}{ccc}
\hline Method & Euclidean distance & SA \\
\hline Physical Model & 0.13 & 0.18 \\
CNN & 0.02 & 0.01 \\
\hline
\end{tabular}

has also confirms the results of Fig. 8, showing the superiority of the proposed CNN over the physical model. The reliance of the physical model on the perfect black and white spots and the fact that they have assumed that the black spot spectrum is independent of wavelength are a few most important reasons why the model does not have a satisfactory output. On the other hand, the CNN makes no presumption and simply learns to model the input image (yellowed image here) to the output image (original colored image) making it a suitable approach to be applied to the problem of virtual cleaning of works of art, in which there are still many unknowns to be answered. One of these is the complexity of the relationship between the paint and the varnish, which needs a far more sophisticated physical model to be understood thoroughly. The need to physically remove a portion of varnish from the painting is also one more impediment in the physical approach making it less suitable to be applied to precious artworks.

\subsection{Color Simulation}

Here we demonstrate the generalizability of the approach to other works of art without any information known about the artwork and the only information available is an RGB image of the uncleaned artwork. In this section, the 500 images in our training samples are all filtered with a yellow filter producing simulated "aged" images. There are three levels of yellowness that could be chosen from; looking at the Mona Lisa and The Virgin and Child with Saint Anne and how yellow they are compared to their cleaned versions, we chose the moderate and high level of yellowness to make the 500 images yellow for The Virgin and Child with Saint Anne and Mona Lisa, respectively. The decision on the level of yellowness of the artworks is made through visual assessment of the work. For example, looking at the color of sky in both paintings here, the sky in the Mona Lisa is much yellower than the sky in The Virgin and Child with Saint Anne. Those images are used to train the 
$\mathrm{CNN}$ to go from the yellowed ("varnished") image to the original ("unvarnished") colored image. Empirically, we note that the closer the images in the training set are to the desired work, in terms of both content and color, the better the results will be. Fig. 9 shows the uncleaned, physically cleaned and virtually cleaned versions of the works used in this paper [15, 35]. The virtually cleaned images are the results of application of the algorithm described here. As shown in Fig. 9, visually, both

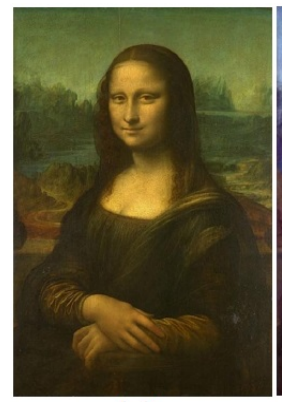

(a)

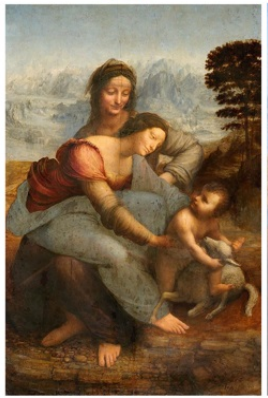

(d)

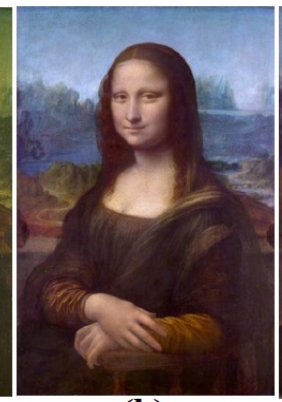

(b)

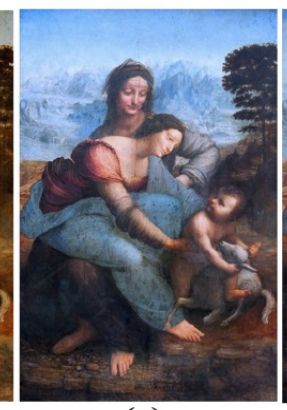

(e)

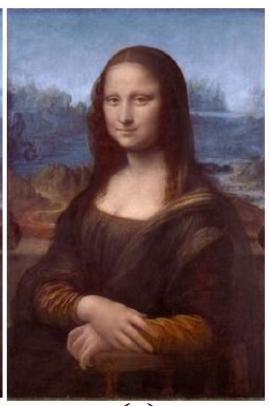

(c)

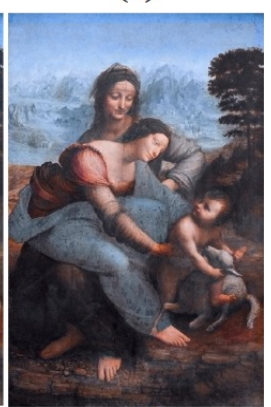

(f)

Figure 9. (a) and (d): Uncleaned, (b) and (e): physically cleaned and (c) and (f): virtually cleaned versions of the works used in this paper.

artworks have been cleaned at a very reasonable level, with The Virgin and Child with Saint Anne having bean cleaned at a higher level compared to Mona Lisa.

To have a better, quantitative understanding of the results, the per-pixel Euclidean distance and SA are computed between the virtually and physically cleaned versions of these works and are shown in Fig. 10. Here, we see that overall, the network has performed better on The Virgin and Child with Saint Anne as compared to the Mona Lisa. This difference shows that the $\mathrm{CNN}$, with a common set of training data, works better on some works than others, likely dependent on many factors. One reason could be that the varnish might not be the only reason for the color change, and the artwork might have experienced some other factors leading to discoloration which would not have been captured in the physical model of the varnish alone. Also, the CNN learns specific features available in the training data. If it does not see a particular feature during training and is then tested on that feature, the $\mathrm{CNN}$ is going to fail. Therefore, the choice of the training samples and how representative they are of the testing data is of great importance. It is hypothesized that the CNN here has learnt two major features, one related to color and the other related to spatial features, such as the sky, the human features, rocks, buildings, and so forth. It is interesting to see that the CNN has done poorly, at least as measured in the Euclidean Distance, on the sky in each image. Again, this is likely a factor of the characteristics of the training data.

By using a Convolutional Neural network with training on simple color images and their artificially yellowed versions, we were able to virtually clean artworks without knowing any information about the works. The only data available to the algorithm is an RGB image of the uncleaned work of art. The results as shown in this paper were satisfactory but for a more generalized network, a great deal of attention should be paid to the datasets used to train the network and also to the level of yellowness of the artwork that are aimed to be virtually cleaned. The work proposed in this paper has two main novelties. The first is the high accuracy of the method, which was proven through drawing a comparison between the method proposed herein with the only physical approach devised until now. The second is the generalizability of the method to different artworks without any information known about the artwork, which is the opposite to all methods devised by now. Having access to the data from at least a few parts of the cleaned and uncleaned art work and not being able to apply the same method devised from one artwork to the other, are two main shortcomings of the prior approaches that are addressed in this work. Having to physically remove the varnish from the artwork is also another negative point from the prior work. However 

works better on realistic looking artworks. Looking at Fig. 1 it is obvious that the network has been trained on the images of humans, buildings and natural subjects making the network more suitable to work on natural (i.e., realistic) images of art. The network likely would not work well on abstract paintings as well as the realistic looking artworks with the training set used here, although that was not tested in this work.

\section{Conclusions}

The problem of virtual cleaning has been of great interest, especially to conservators and curators in museums, which could help them visualize how a painting would change with cleaning. Many approaches have been developed and applied to solve this problem, most of which rely on the physical removal of the varnish from different parts of the painting. Reliance on finding black and white spots on the painting is another shortcoming of these approaches. In order to circumvent this problem, a Convolutional Neural Network (CNN) was applied to the issue of virtual cleaning of works of art. In order to do that, an RGB picture of paintings were artificially yellowed using physically-derived yellow filters and the network was trained to go from these yellowed images to the original colored ones. Comparing our work with a physical model showed the superiority of the method proposed in this paper to the physical approach used to virtually clean the works of art. The method was applied to two famous works of art, namely, the Mona Lisa and The Virgin and Child with Saint Anne, leading to a satisfactory virtual cleaning of bothw. The main two novelties of the method proposed herein are the high accuracy, proven through comparing the method to the only physical based approach and the generalizability of it, which was shown through applying the method blindly to the two famous works of art, for which the only piece of information available is the uncleaned RGB image of work of art. One of the limitation of the network trained herein is that it does not work as well on the abstract type of paintings, as it has been trained on the realistic looking images.

\section{Abbreviations}

CNN: Convolutional Neural Network; RGB: Red, Green and Blue color space; CIE: Commission Internationale de l'Eclairage;CIEXYZ: X, Y and Z color space approved by CIE; sRGB: standard RGB color space; JPEG: Joint Photographic Experts Group, digital image compression method; SA: Spectral Angle. 


\section{Authors' Contributions}

MMA came up with the idea, coded up and trained and tested the CNN and applied it to the data. DWM oversaw and advised the research; Both authors wrote the article and approved of the final version of the manuscript.

\section{Funding}

The work was done as a PhD research and supported by Rochester Institute of Technology.

\section{Availability of the Data and Materials}

The datasets used and/or analysed during the current study are available from the corresponding author on reasonable request.

\section{Competing interests}

The authors declare that they have no competing interests.

\section{Author details}

Chester F. Carlson Center for Imaging Science, Rochester Institute of Technology, Lomb Memorial Drive, 14623 Rochester, NY, USA.

\section{References}

[1] S. Constantin, "The barbizon painters: a guide to their suppliers," Studies in conservation, vol. 46, no. 1, pp. 49-67, 2001.

[2] A. Callen, “The unvarnished truth: Mattness,'primitivism'and modernity in french painting, c. 1870-1907," The Burlington Magazine, vol. 136, no. 1100, pp. 738-746, 1994.

[3] R. Bruce-Gardner, G. Hedley, and C. Villers, "Impressionist and post-impressionist masterpieces: The courtauld collection," 1987.

[4] M. Watson and A. Burnstock, "An evaluation of color change in nineteenth-century grounds on canvas upon varnishing and varnish removal," in New Insights into the Cleaning of Paintings: Proceedings from the Cleaning 2010 International Conference, Universidad Politecnica de Valencia and Museum Conservation Institute, Smithsonian Institution, 2013.

[5] R. S. Berns and E. R. De la Rie, "The effect of the refractive index of a varnish on the appearance of oil paintings," Studies in conservation, vol. 48, no. 4, pp. 251-262, 2003.

[6] L. V. Angelova, B. Ormsby, J. Townsend, and R. Wolbers, Gels in the Conservation of Art. Archetype Publications, 2017.

[7] B. Lambert, H. Joen, O. Bronwyn, N. Petria, I. Piet, and K. Katrien, "A review of solvent action on oil paint," Heritage Science, vol. 8, no. 1, 2020.

[8] S. Prati, F. Volpi, R. Fontana, P. Galletti, L. Giorgini, R. Mazzeo, L. Mazzocchetti, C. Samorì, G. Sciutto, and E. Tagliavini, "Sustainability in art conservation: a novel bio-based organogel for the cleaning of water sensitive works of art," Pure and Applied Chemistry, vol. 90, no. 2, pp. 239-251, 2018.

[9] E. Al-Emam, H. Soenen, J. Caen, and K. Janssens, "Characterization of polyvinyl alcohol-borax/agarose (pva-b/ag) double network hydrogel utilized for the cleaning of works of art," Heritage Science, vol. 8, no. 1, pp. 1-14, 2020.

[10] M. El-Gohary, "Experimental tests used for treatment of red weathering crusts in disintegrated granite-egypt," Journal of cultural heritage, vol. 10, no. 4, pp. 471-479, 2009.

[11] D. Gulotta, D. Saviello, F. Gherardi, L. Toniolo, M. Anzani, A. Rabbolini, and S. Goidanich, "Setup of a sustainable indoor cleaning methodology for the sculpted stone surfaces of the duomo of milan," Heritage Science, vol. 2, no. 1, p. 6, 2014. 
[12] G. Trumpy, D. Conover, L. Simonot, M. Thoury, M. Picollo, and J. K. Delaney, "Experimental study on merits of virtual cleaning of paintings with aged varnish," Optics express, vol. 23, no. 26, pp. 33836-33848, 2015.

[13] M. Barni, F. Bartolini, and V. Cappellini, "Image processing for virtual restoration of artworks," IEEE multimedia, vol. 7, no. 2, pp. 34-37, 2000.

[14] M. Pappas and I. Pitas, "Digital color restoration of old paintings," IEEE Transactions on image processing, vol. 9, no. 2, pp. 291-294, 2000.

[15] M. Elias and P. Cotte, "Multispectral camera and radiative transfer equation used to depict leonardo's sfumato in mona lisa," Applied optics, vol. 47, no. 12, pp. 2146-2154, 2008.

[16] C. M. T. Palomero and M. N. Soriano, "Digital cleaning and dirt layer visualization of an oil painting," Optics express, vol. 19, no. 21, pp. 21011-21017, 2011.

[17] L. Yang and B. Kruse, "Revised kubelka-munk theory. i. theory and application," JOSA A, vol. 21, no. 10, pp. 19331941, 2004.

[18] E. Kirchner, I. van der Lans, F. Ligterink, E. Hendriks, and J. Delaney, "Digitally reconstructing van gogh's field with irises near arles. part 1: varnish," Color Research \& Application, vol. 43, no. 2, pp. 150-157, 2018.

[19] J. Linhares, L. Cardeira, A. Bailão, R. Pastilha, and S. Nascimento, "Chromatic changes in paintings of adriano de sousa lopes after the removal of aged varnish," Conservar Património, vol. 34, pp. 50-64, 2020.

[20] L. M. Dang, S. I. Hassan, S. Im, and H. Moon, "Face image manipulation detection based on a convolutional neural network," Expert Systems with Applications, vol. 129, pp. 156-168, 2019.

[21] S. Ratnasingam, "Deep camera: A fully convolutional neural network for image signal processing," in Proceedings of the IEEE International Conference on Computer Vision Workshops, pp. 0-0, 2019.

[22] Y. Zhang, Y. Liu, P. Sun, H. Yan, X. Zhao, and L. Zhang, "Ifcnn: A general image fusion framework based on convolutional neural network," Information Fusion, vol. 54, pp. 99-118, 2020.

[23] S. S. Yadav and S. M. Jadhav, "Deep convolutional neural network based medical image classification for disease diagnosis," Journal of Big Data, vol. 6, no. 1, p. 113, 2019.

[24] R. S. Shankar, G. Mahesh, K. Murthy, and D. Ravibabu, "A novel approach for gray scale image colorization using convolutional neural networks," in 2020 International Conference on System, Computation, Automation and Networking (ICSCAN), pp. 1-8, IEEE, 2020.

[25] R. Zhang, P. Isola, and A. A. Efros, "Colorful image colorization," in European conference on computer vision, pp. 649666, Springer, 2016.

[26] R. Dhir, M. Ashok, S. Gite, et al., "An overview of advances in image colorization using computer vision and deep learning techniques," Review of Computer Engineering Research, vol. 7, no. 2, pp. 86-95, 2020.

[27] S. Anwar, M. Tahir, C. Li, A. Mian, F. S. Khan, and A. W. Muzaffar, "Image colorization: A survey and dataset," arXiv preprint arXiv:2008.10774, 2020.

[28] DanB, “Urban and rural photos,” 062018.

[29] J. P. Parkkinen, J. Hallikainen, and T. Jaaskelainen, "Characteristic spectra of munsell colors," JOSA A, vol. 6, no. 2, pp. 318-322, 1989.

[30] R. S. Berns, Billmeyer and Saltzman's principles of color technology. John Wiley \& Sons, 2019.

[31] Y. Okumura, "Developing a spectral and colorimetric database of artist paint materials," 2005.

[32] A. Gulli and S. Pal, Deep learning with Keras. Packt Publishing Ltd, 2017. 
[33] H. Wu, X. Sun, J. Yang, W. Zeng, and F. Wu, "Lossless compression of jpeg coded photo collections," IEEE Transactions on Image Processing, vol. 25, no. 6, pp. 2684-2696, 2016.

[34] B. Park, W. Windham, K. Lawrence, and D. Smith, "Contaminant classification of poultry hyperspectral imagery using a spectral angle mapper algorithm,” Biosystems Engineering, vol. 96, no. 3, pp. 323-333, 2007.

[35] D. Bair, "Leonardo da Vinci discovering da vinci," 2010. 


\section{Figures}
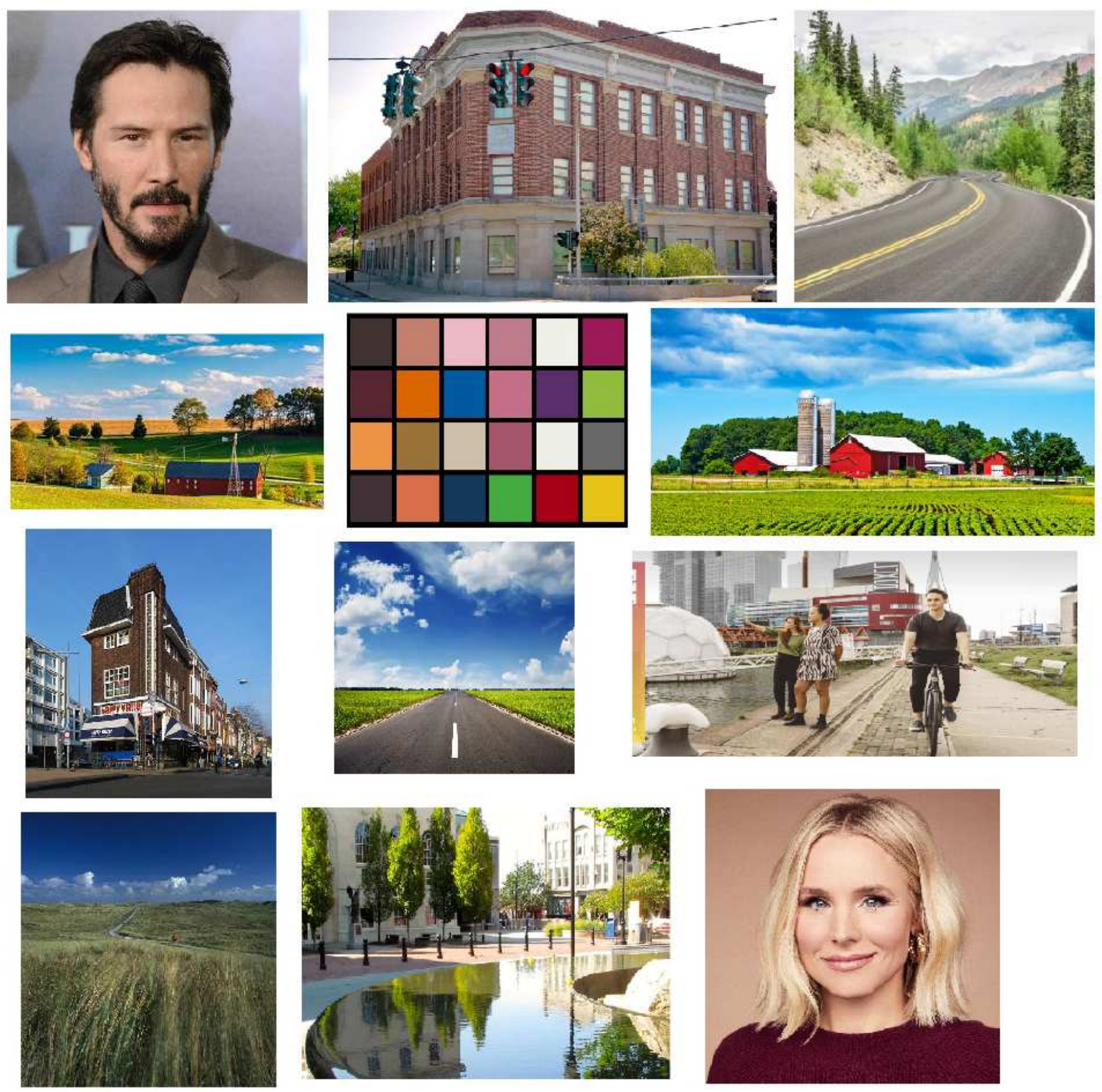

Figure 1

Some examples of the images used for training in this work. 


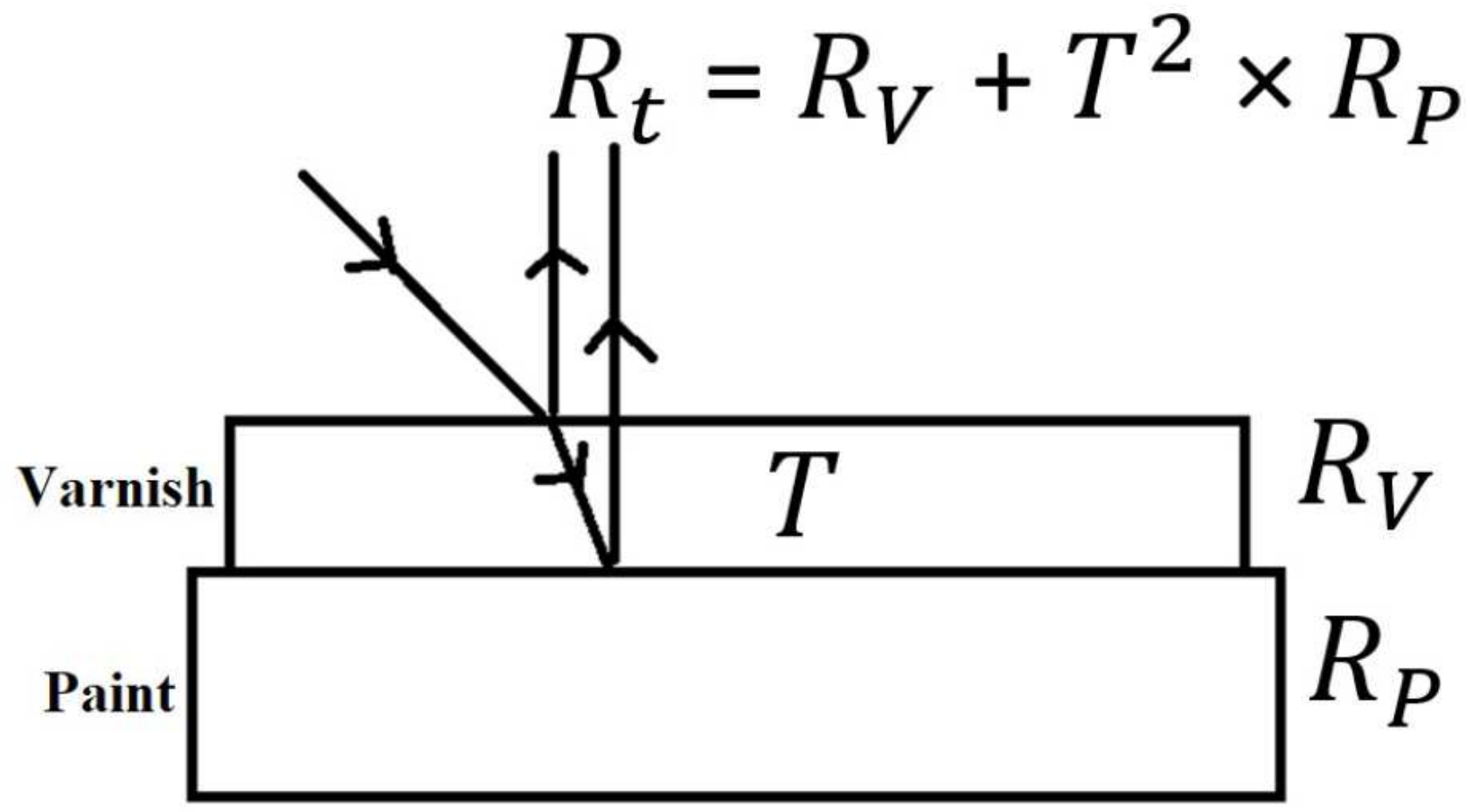

Figure 2

Interaction of light with varnish/painting system.

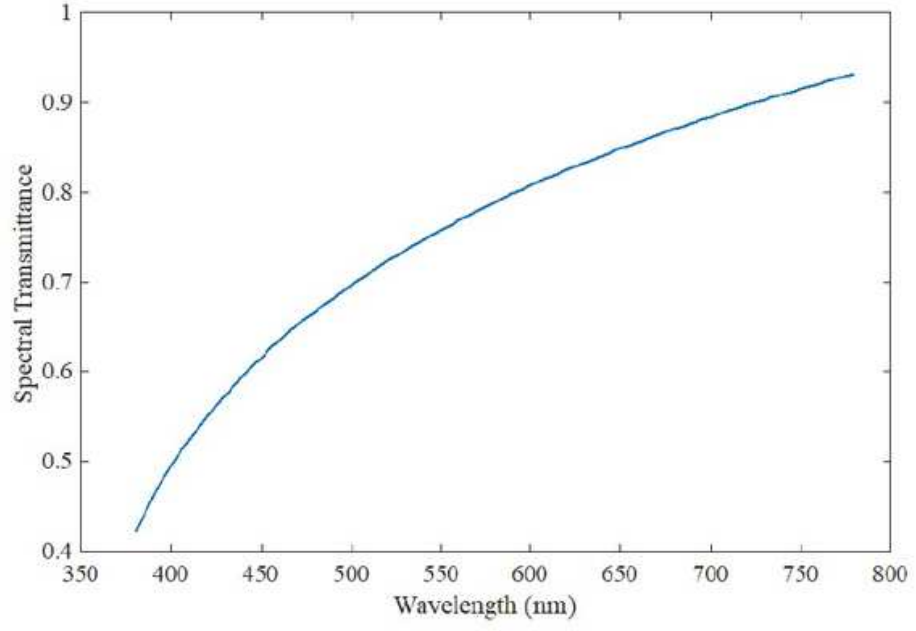

(a)

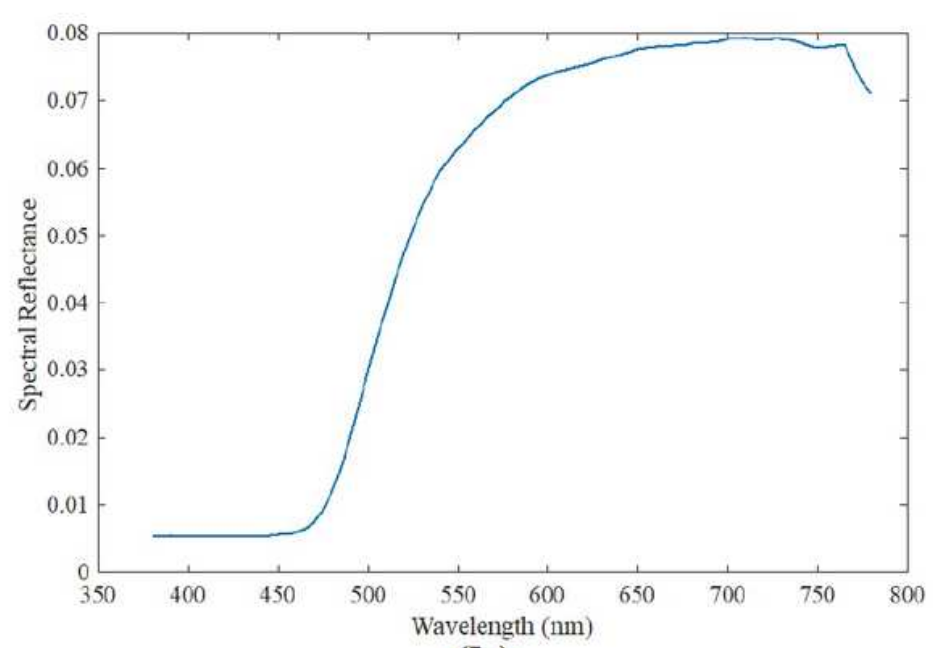

(b)

Figure 3

(a) Transmittance and (b) reflectance spectra of the varnish. 


\section{(a)}

(b)

(c)

Figure 4

Yellow filters at three levels used to artificially yellow the images.

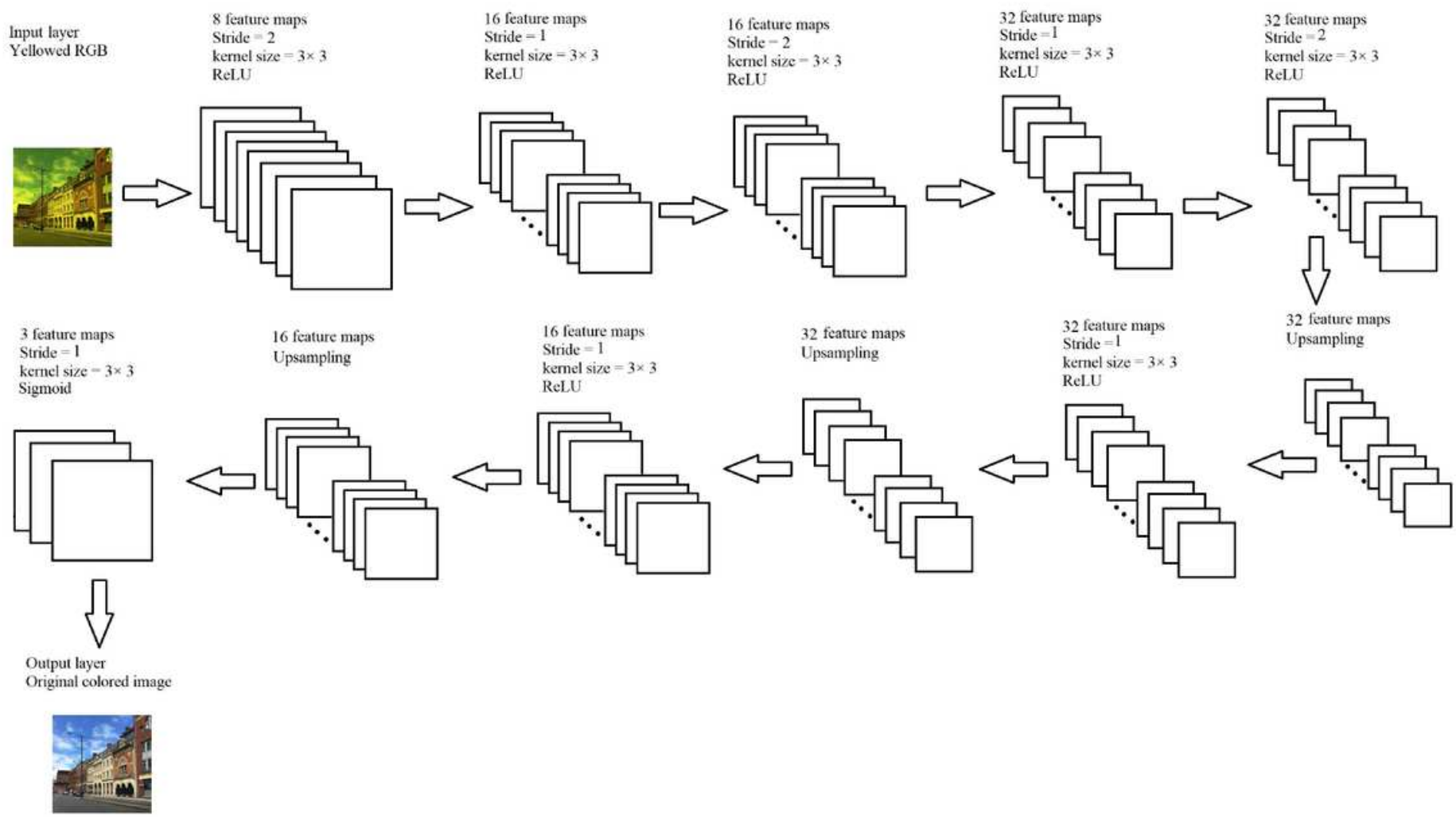

Figure 5

Architecture of the CNN used in this work. 


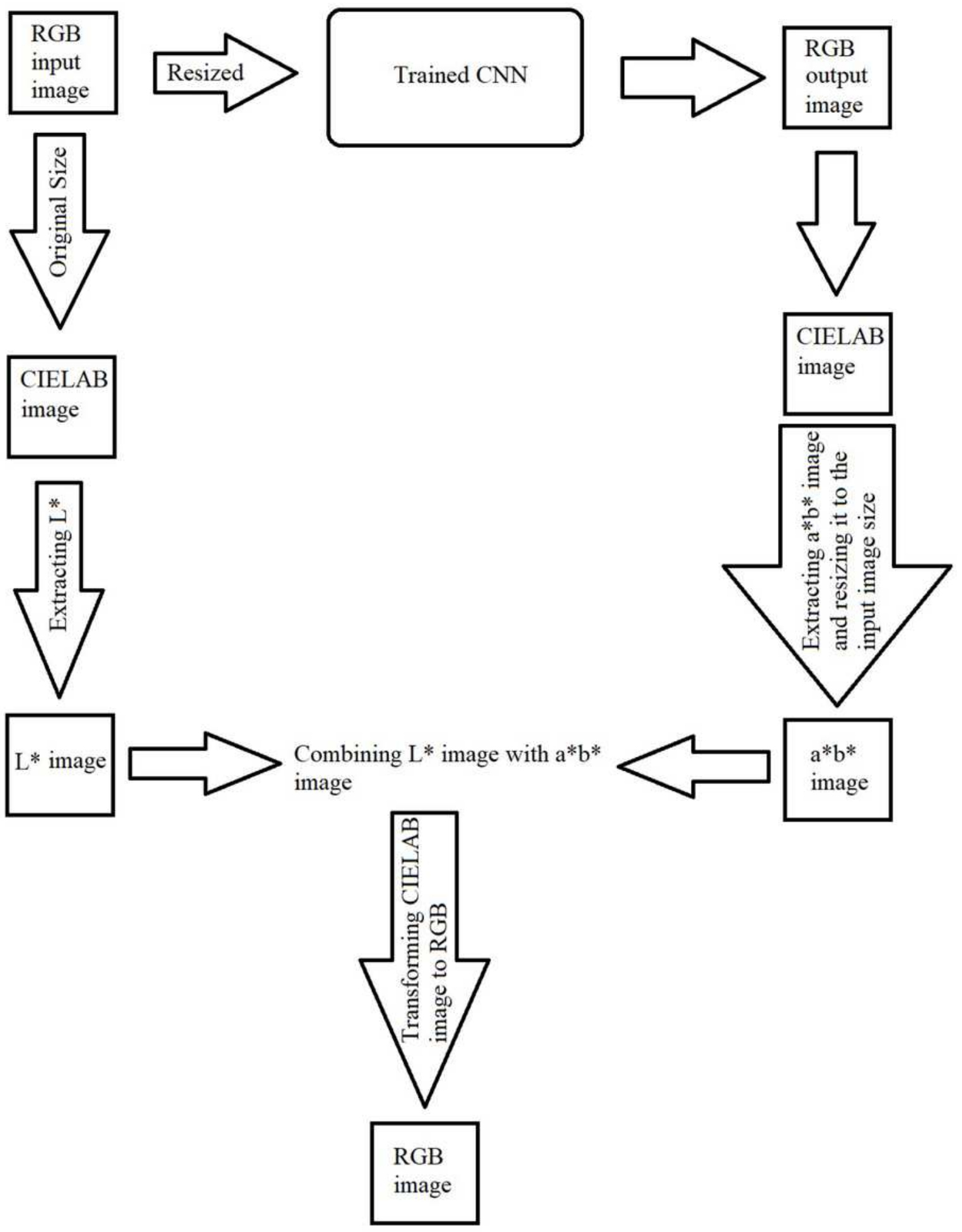

Figure 6

Yellow filters at three levels used to artificially yellow the images. 

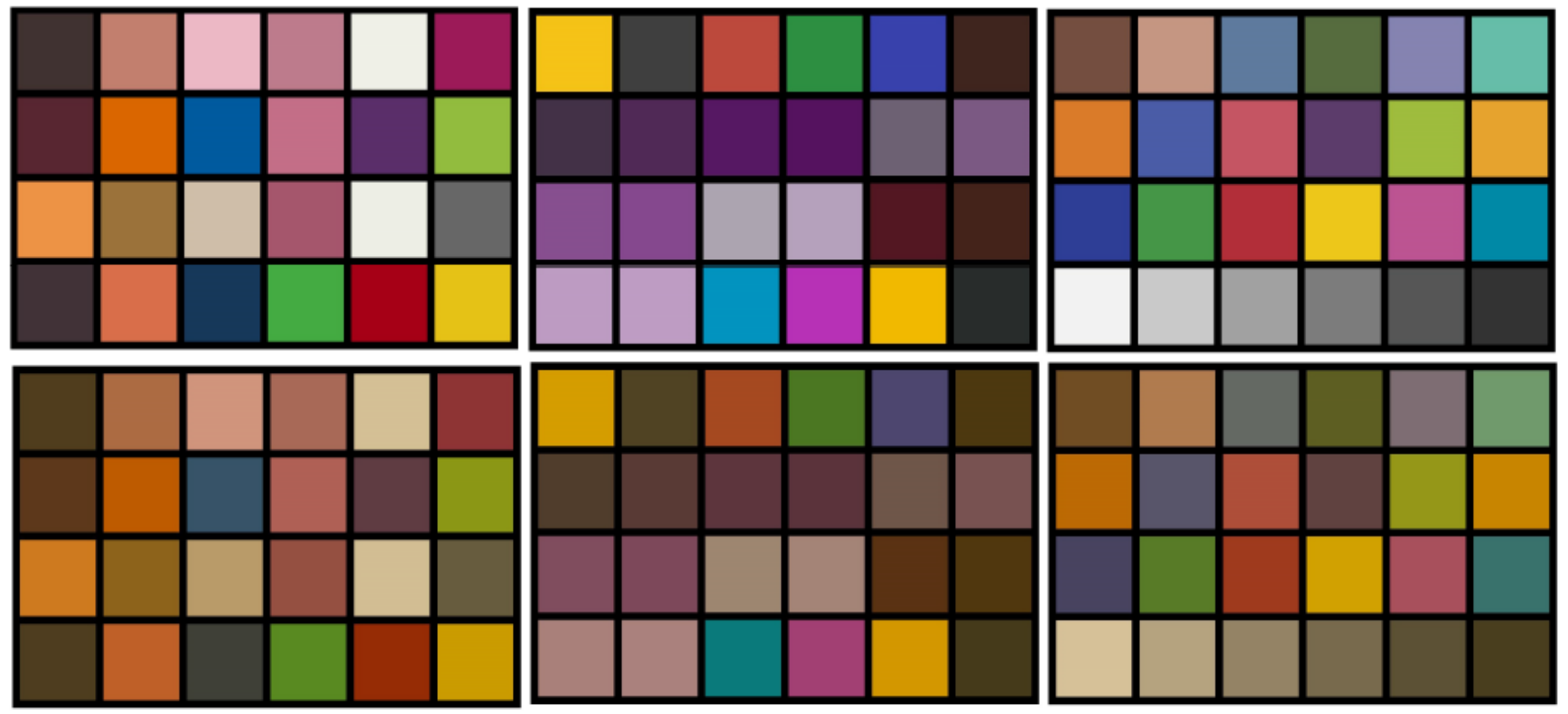

Figure 7

Simulated color charts (top row) and their yellowed versions (bottom row). 


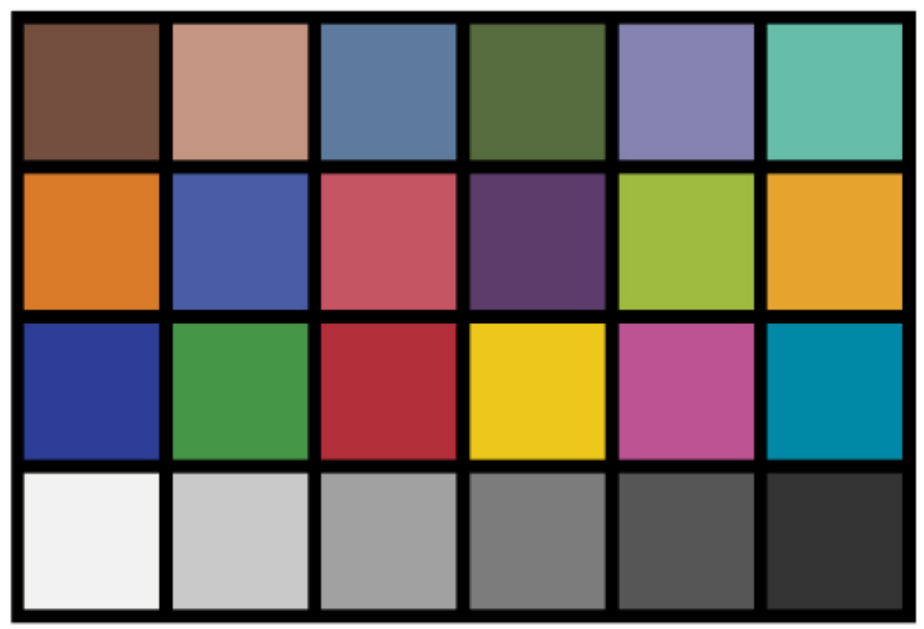

(a)

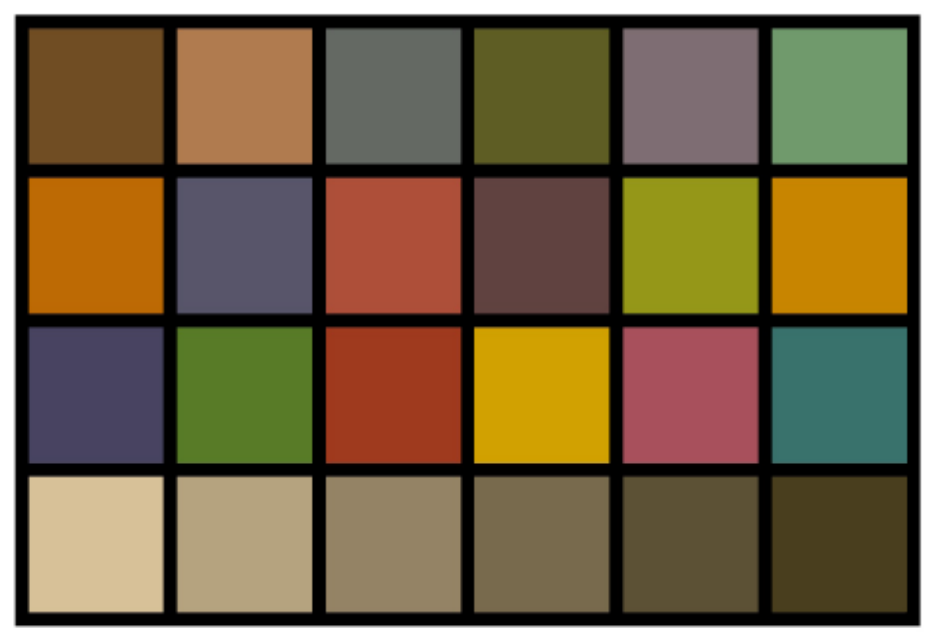

(c)

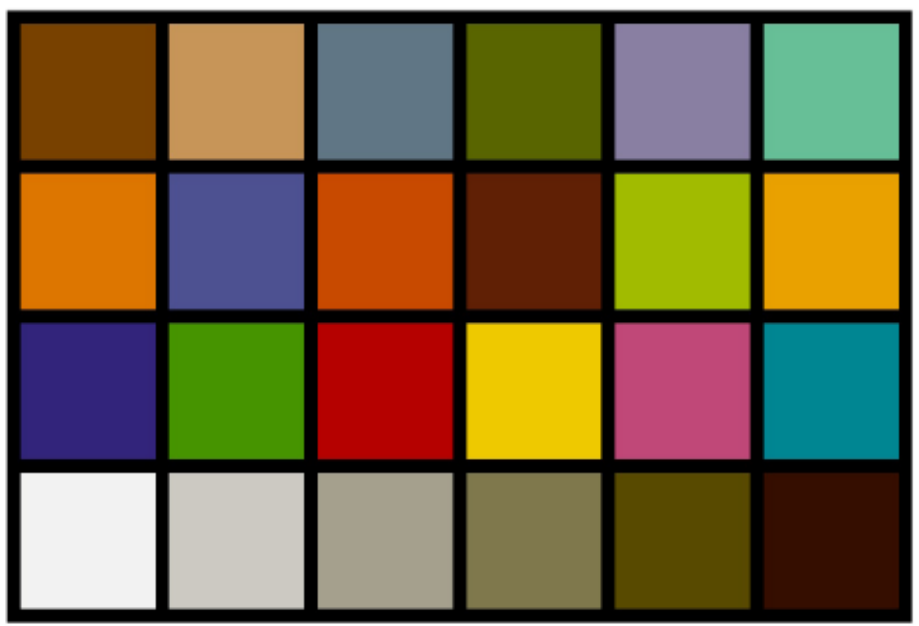

(b)

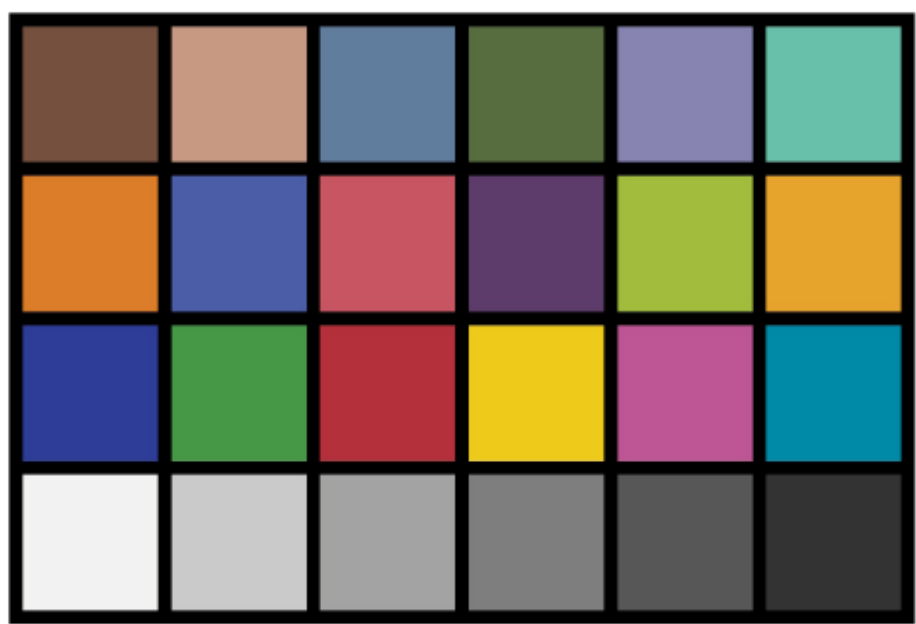

(d)

Figure 8

a: original Macbeth, b: physical model output, c: yellowed Macbeth, d: CNN output. 


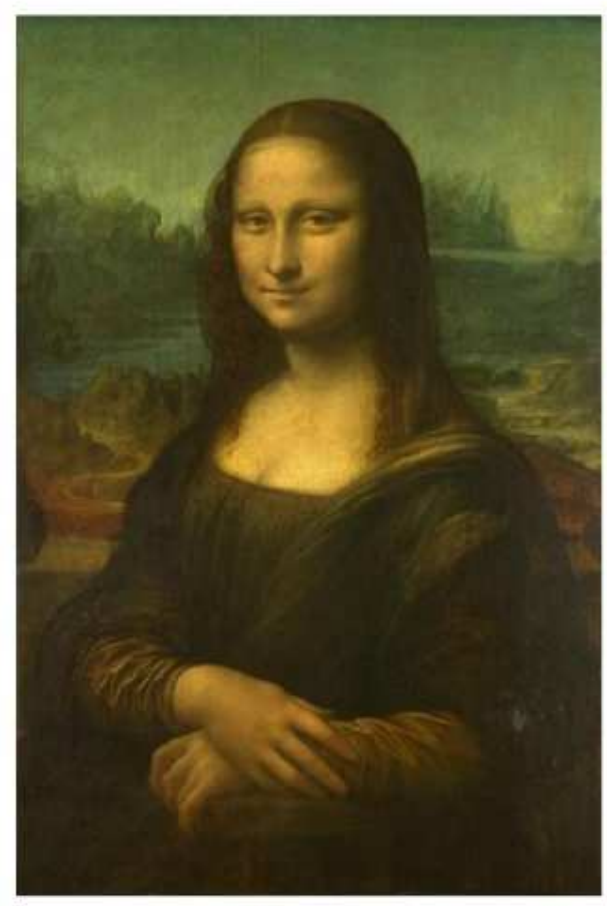

(a)

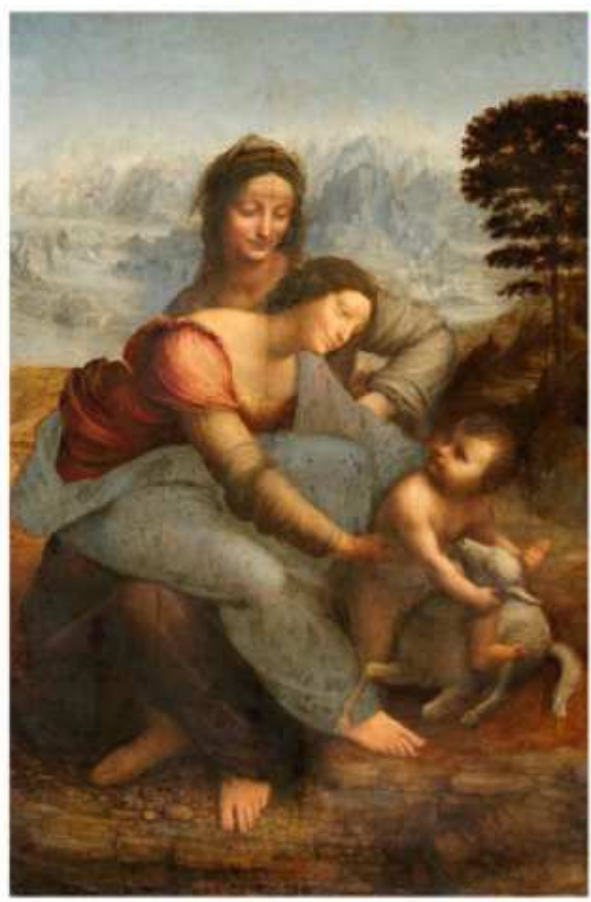

(d)

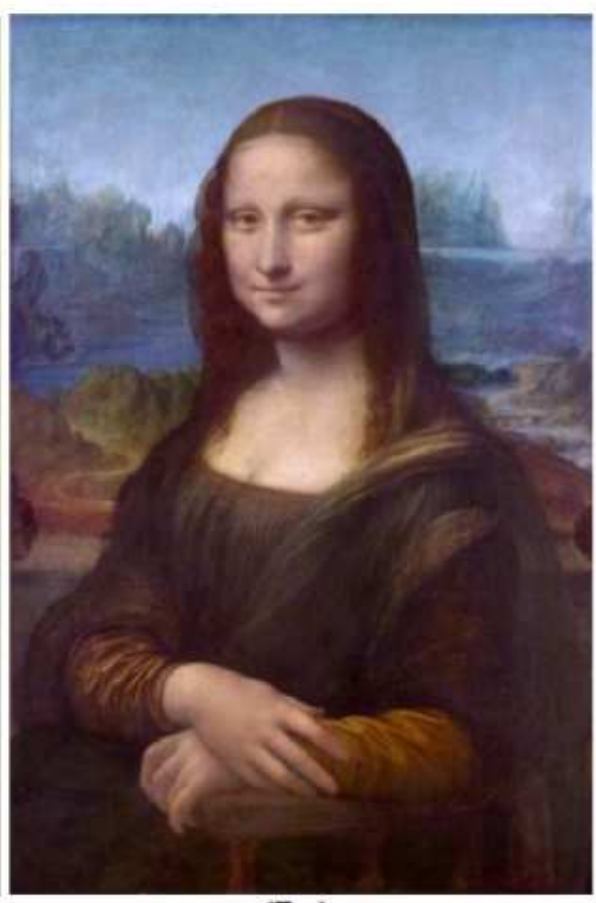

(b)

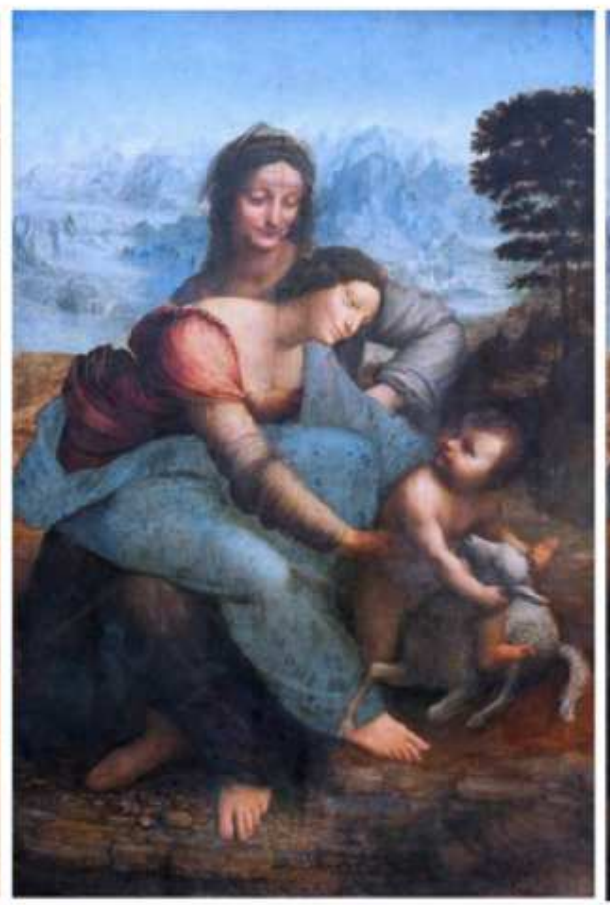

(e)

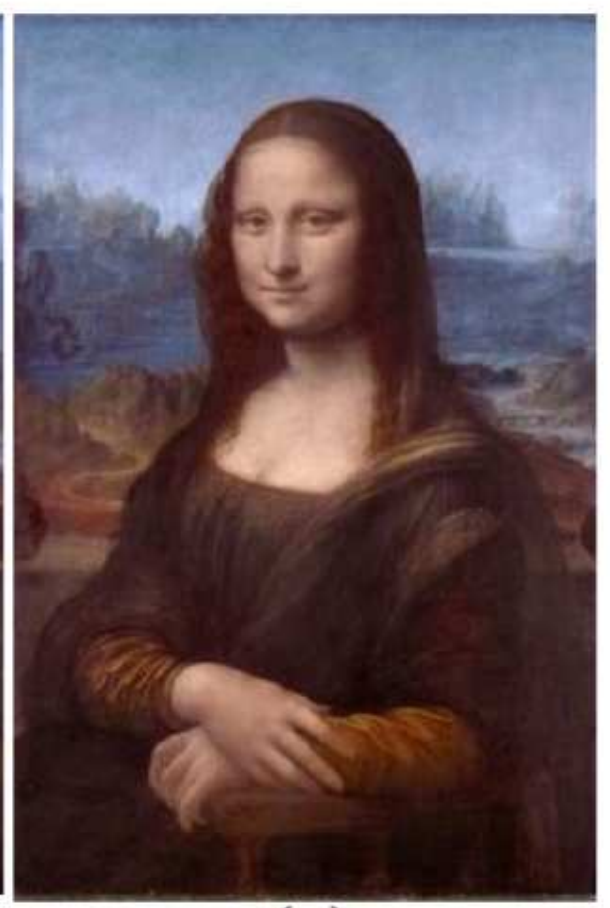

(c)

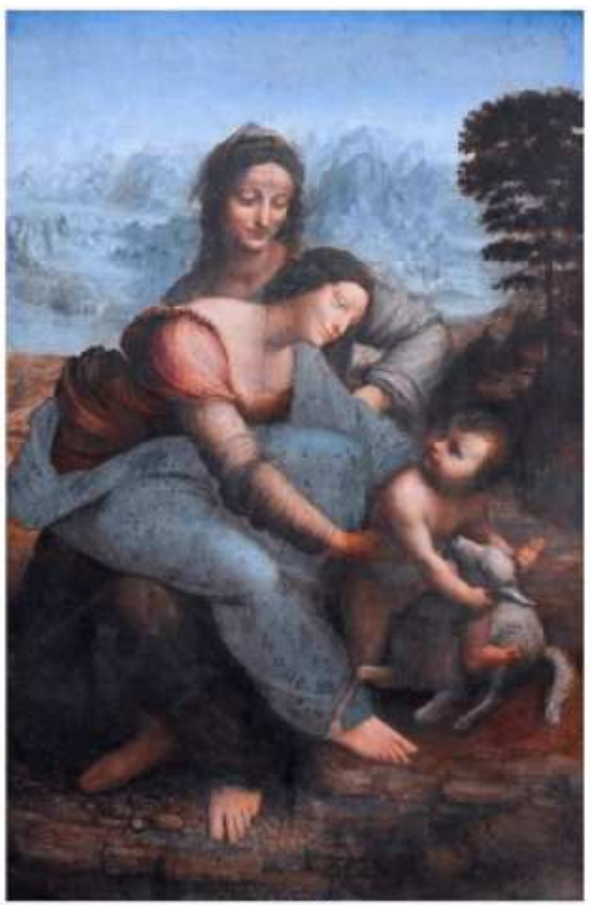

(f)

\section{Figure 9}

(a) and (d): Uncleaned, (b) and (e): physically cleaned and (c) and (f): virtually cleaned versions of the works used in this paper. 


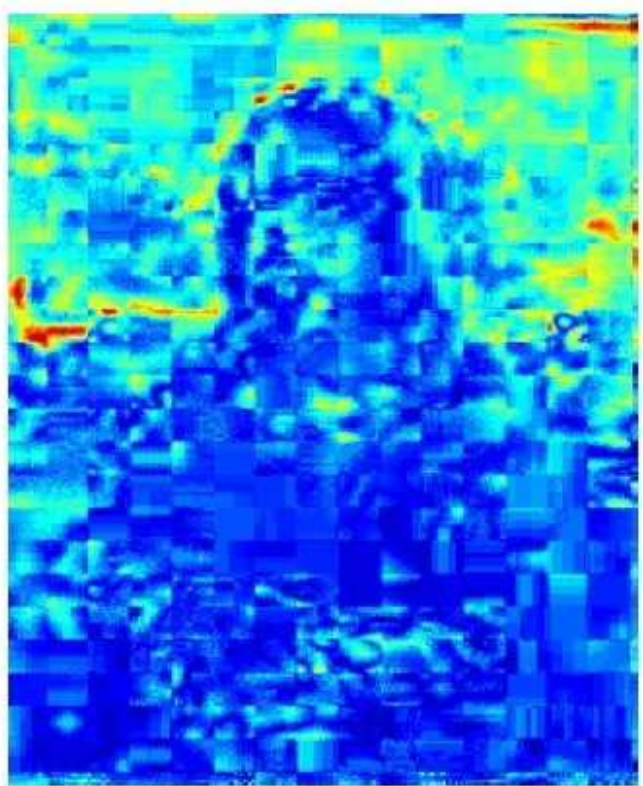

(a)

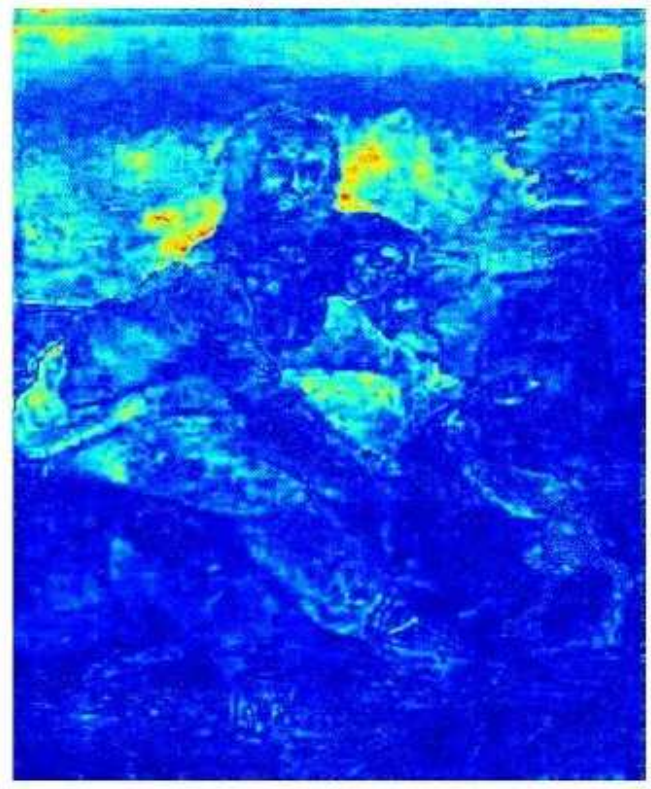

(c)

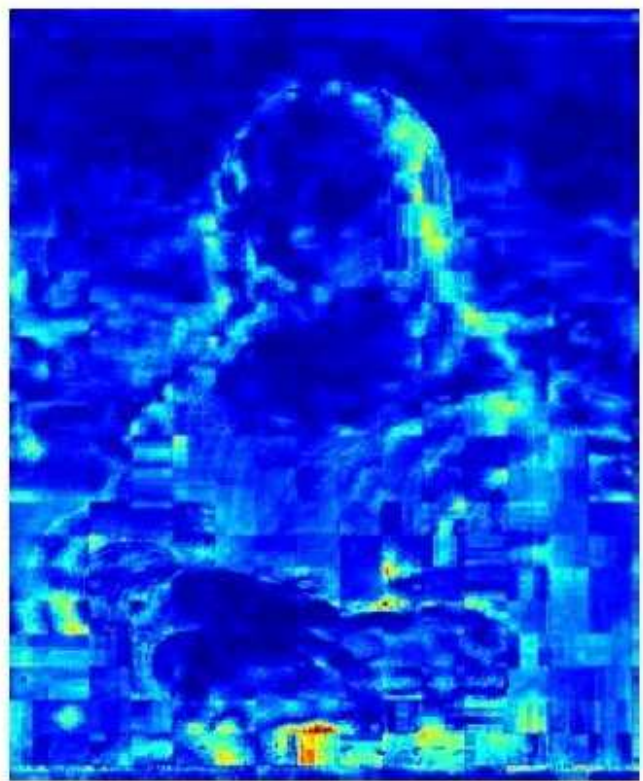

(b)

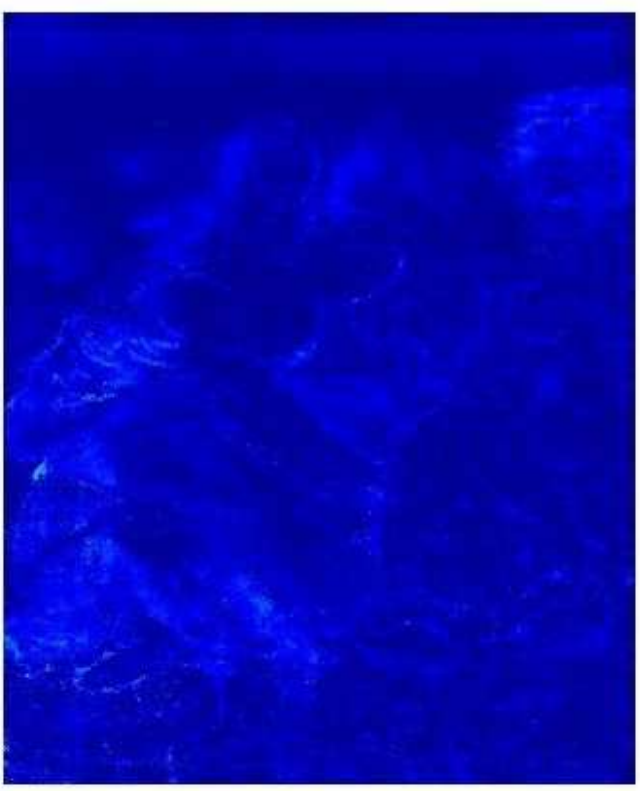

(d)

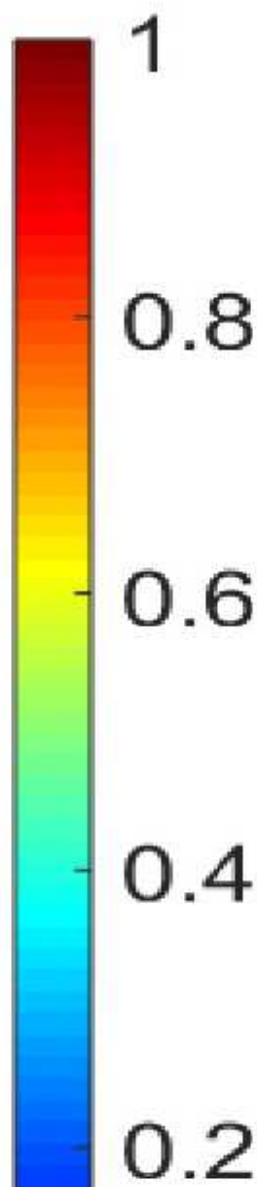

0.2

0

Figure 10

Euclidean distance (left column) and SA (right column) computed between the virtually and physically cleaned versions of the Mona Lisa, (a) and (b), and The Virgin and Child with Saint Anne, (c) and (d). 